\title{
Central Neck Dissection in Papillary Thyroid Cancer Allows for Avoidance of Radioactive Iodine
}

Alexander T. Reid, BA; James H. Hammond, BS; Joshua D. Smith, BA; Adam C. Niemann, BS; David T. Hughes, MD

\section{ABSTRACT}

\section{Background}

Prophylactic central neck dissection (pCND) is an adjunct to total thyroidectomy (TT) for papillary thyroid cancer (PTC) and allows detection of occult lymph node metastasis. Our hypothesis is that pCND does not increase the use of radioactive iodine (RAI) therapy.

\section{Methods}

This is a retrospective cohort study of patients who underwent $\Pi$ alone or $\Pi$ with pCND for PTC. Patient demographics, histopathological features (capsular invasion, lymph node metastasis, vascular invasion, extranodal extension, positive margin), RAl use, and RAI dose were compared between $T T$ al one and $\Pi T$ with pCND groups.

\section{Results}

$25 \pi$ and 73 TT with pCND proCorresponding Author: Alexander Reid, BA, alexreid @umich.edu, 605-645-7804

Conflicts of interest: The authors have no conflicts of interest to disclose.

\section{Introduction}

with pCND cohort was younger (45 vs 53; $p=0.024$ ) and had more capsular invasion (33 [45\%] vs 5 [20\%], $p=0.032$ ) and more lymph node metastasis (24 [33\%] vs $1[4 \%], p=0.003)$. There was no significant difference in the number of patients receiving RAI therapy $(p=1.000)$ or the RAI dose ( $p=0.176$ ) between the $\Pi$ and $\Pi$ with $\mathrm{PCND}$ groups. Separating the $\Pi T$ with pCND group into NO and N1a cohorts revealed that N1a patients received RAI therapy more frequently than NO patients (24 [100\%] vs 30 [61\%], $p=0.0001)$.

\section{Conclusions}

TT with PCND for PTC does not cause an increase in upstaging patients and thereby lead to an increase in the use of RAl therapy.

he incidence of papillary thyroid cancer (PTC) continues to increase with nearly 40,000 cases occurring in the United States in 2015. 'Total thyroidectomy (TT) and radioactive iodine (RAI) 
therapy are highly effective treatments for PTC, providing patients with 10-year survival rates over 90\%. ${ }^{2,3}$ Cervical lymph node metastases are common in PTC patients. Lymph node involvement is histologically found in $37 \%$ to $64 \%$ of PTC patients, even if they didn't have preoperative evidence of lymph node involvement. ${ }^{2,4}$ The central neck compartment nodes (Level VI nodes) are the most common site of PTC metastasis. This contributed to the idea of sampling these nodes by performing a prophylactic central neck dissection (pCND) in addition to a total thyroidectomy (TT) in order to guide post-operative treatment., ${ }^{2,3} T$ with $\mathrm{pCND}$ remains controversial due to its uncertain effect on survival, recurrence rates, and effect on RAI therapy compared to TT alone. ${ }^{1}$

Nodal staging in PTC treated with $\mathrm{TT}$ alone is incomplete since the central node compartments are not sampled. Recommendations for further treatment, typically RAI therapy, rely solely on the pathologic characteristics of the primary tumor, the presence or absence of distant metastasis, and the patient's demographics. The American Thyroid Association (ATA) 2015 guidelines rely heavily on risk stratification of PTC based on surgical pathology, which includes nodal stage 1.1

Acute complications of RAl therapy include post-treatment 24-hour isolation to reduce radiation exposure to others, nausea vomiting, and tumor hemorrhage.5,6 Longer term consequences include sialadenitis resulting in xerostomia, mouth pain, dental caries, pulmonary fibrosis, and nasolacrimal outflow obstruction, as well as secondary malignancies, namely breast carcinoma, leukemia, and salivary gland cancer.7,8

Whether TT with pCND can be used to determine the appropriateness of post-operative RAI therapy remains uncertain. In cases of micropapillary carcinoma (tumor size $<1 \mathrm{~cm}$ ) it may be that RAI therapy is not indicated. ${ }^{9}$ However, our study excludes this specific group. Randolph argues that TT with pCND upstages roughly 35\% of patients, leading to unnecessary RAl therapy with its associated aforementioned risks. ${ }^{10}$ Others, including
Sosa, have found that TT with PCND decreased unnecessary RAI therapy. ${ }^{11-14}$

Bonnet et al studied pCND in the context of tumors smaller than $2 \mathrm{~cm} .{ }^{15}$ Our work examines larger tumor size, which is a worse prognostic feature and may affect the decision to use RAI therapy. Hughes et al performed TT with pCND research that focused on surgical outcomes and RAl dosage. ${ }^{16}$ Our study population includes more recent data when newer ATA guidelines were in place. Additionally, we examine differences in the number of patients within the $\Pi$ with $p C N D$ group that received RAI therapy, not just differences in RAI dose. Our study is important because current literature is mixed as to the appropriateness of $\Pi$ with pCND, and our paper will offer additional insight into this dilemma. Our hypothesis is that $\Pi$ with pCND will not increase the use of RAl therapy compared to $\Pi$ alone but will direct RAI therapy toward higher-risk, node-positive patients and away from lower-risk, node-negative patients.

\section{Methods Study Population}

Following approval from the University of Michigan Institutional Review Board (IRB), we performed a retrospective analysis of patients having $T T$ alone or $T T$ with pCND for papillary thyroid cancer (2006-2012). The institutional database contained 820 patients from 2006-2012. Seven hundred twenty-two patients were excluded for the following reasons: surgical procedure other than a $T T$ or $\Pi T$ with $\mathrm{pCND}$, preoperative evidence of lymph node metastasis or local tumor invasion, non-papillary thyroid cancer or high-risk variant PTC, papillary microcarcinoma (tumor size $<1 \mathrm{~cm}$ ), presence of distant metastasis, presence of lateral neck metastasis on surgical pathology (N1 b), presence of local tumor invasion on surgical pathology (T4), or incomplete medical records. Ninety-eight patients met inclusion criteria and were analyzed by primary surgical procedure: $\pi$ or $\Pi \pi$ with pCND.

The surgeon made the decision to perform a $\Pi$ with pCND based on preoperative assessment of 
the patient (age, family history, radiation exposure, other risk factors) and the primary tumor on exam and imaging (size of tumor, suspicion for extrathyroidal extension, contralateral nodularity). At the time of the study, the 2009 ATA guidelines recommended $\Pi$ with pCND (albeit with weak evidence) and, therefore, the surgeons followed these guidelines. ${ }^{18} \mathrm{~A} T \mathrm{~T}$ alone may also have been performed because preoperatively it was unclear if the cancer was PTC, for example, when a previous indeterminate Fine Needle Aspiration (FNA) was performed or the PTC was found incidentally when the $\Pi T$ was done for another indication (multinodular goiter, most commonly).

Central neck dissection was defined as removal of nodes found in level $\mathrm{VI}$, including prelaryngeal, pretracheal, and paratracheal lymph nodes. ${ }^{3}$ A subgroup analysis of $\Pi$ with $\mathrm{pCND}$ patients with regard to nodal status (NO vs N1a) was also performed. Clinicopathological variables, including patient age, sex, tumor size, capsular invasion, vascular invasion, and extranodal extension, were collected based on American Joint Committee on Cancer (AJCC) staging criteria. ${ }^{1}$ Lymph node metastasis was determined histologically, not with polymerase chain reaction.

AJCC criteria for tumor, node, metastasis (TNM) classification of differentiated thyroid carcinoma classifies N0 as no metastatic lymph nodes, N1a as metastasis to level VI (pre-para-tracheal and prelaryngeal nodes), and N1b as metastasis to cervical or superior mediastinal nodes. M0 signifies no distant metastasis, and M1 indicates distant metastasis. $\mathrm{T1}$ is primary tumor diameter of $2 \mathrm{~cm}$ or smaller, T2 is tumor size $>2-4 \mathrm{~cm}, \mathrm{~T} 3$ is tumor size $>4 \mathrm{~cm}$, and T4 is determined by a high degree of invasion to nearby subcutaneous tissue or fascia. Stage I is defined as patients younger than 45 years old without evidence of distant metastasis (M0), while stage II does have evidence of distant metastasis (M1). For patients 45 years or older, stage I is T1,N0,MO; stage II is T2,NO,M0; stage III is T3,N0,M0; and stage IV is T1-3 with N1a, T4 with any $\mathrm{N}$, or $\mathrm{M} 1$ with any $\mathrm{T}$ and any $\mathrm{N} .{ }^{1}$

ATA 2006 and 2009 guidelines were followed to determine RAI use during the study period. The
ATA 2006 guidelines recommend RAI therapy for stage IV disease; stage II and stage III disease for patients younger than 45 years; stage II disease for most patients older than 45 years; and select stage I patients with "multifocal disease, nodal metastases, extrathyroidal or vascular invasion and/ or more aggressive histologies."17 ATA 2009 guidelines state that "RAI ablation is recommended for selected patients with 1-4 cm thyroid cancers confined to the thyroid who have documented lymph node metastases or other higher risk features when the combination of age, tumor size, lymph node status, and individual histology predicts an intermediate to high risk of recurrence or death from thyroid cancer."199 The decision to use RAl therapy in our study was based on the clinical judgement of individual surgeons, endocrinologists, and nuclear medicine physicians using the aforementioned guidelines, as well as RAI scans, presence of thyroglobulin antibodies, and pre-op thyroglobulin levels. RAl therapy and dose were compared between patients who underwent $\Pi$ alone and those who underwent $\Pi T$ with $\mathrm{pCND}$.

\section{Statistical Analysis}

The primary outcomes were RAI therapy use and dose of RAl following $\Pi T$ alone or $\Pi$ with $\mathrm{pCND}$, as well as surgical outcomes, including laryngeal nerve injury, permanent hypoparathyroidism, and symptomatic hypocalcemia. The relationships between clinicopathologic variables and RAI therapy or RAI dose were considered significant if the $p$ value was less than 0.05. The significance of clinicopathological variables was calculated using Fisher's exact test for categorical variables, Mann-Whitney $U$, or Student T-test for continuous variables. Prism GraphPad was used for all statistical analysis.

\section{Results}

$\Pi$ and $\Pi$ with $\mathrm{pCND}$ demographic data and postoperative tumor characteristics are compared in Table 1. The $\pi$ alone group ( $n=25$ ) was significantly older than the $\Pi$ with pCND group $(n=73)$ (52.6 years vs 45 years, $p=0.024)$. The $\Pi$ with $p C N D$ group had 
significantly more capsular invasion (33 [45\%] vs 5 [20\%], $p=0.032$ ) and more lymph node metastasis (24 [33\%] vs 1 [4\%], $p=0.003$ ). The single patient with lymph node metastasis in the $\Pi$ alone cohort was due to a positive peri-thyroidal lymph node that was resected with the thyroid specimen. There were no significant differences in sex $(p=0.588)$, median tumor size $(p=0.434)$, vascular invasion $(p=0.271)$, extranodal extension ( $p=0.568$ ), or positive margins ( $p=0.280$ ) between the $\Pi$ alone and $\Pi$ with pCND groups. There was no difference in RAI therapy received between the $\Pi$ only and $\Pi \pi$ with $p C N D$ cohorts (18 [72\%] vs 54 [74\%], $p=1.000)$ and no difference in mean RAI dose between the $\Pi$ alone and $\Pi$ with pCND cohorts (62 millicuries vs 77 millicuries, $p=0.176)$.

TABLE 1. Clinicopathological variables and Radioactive lodine (RAl) between Total Thyroidectomy (TT) alone and Total Thyroidectomy with Prophylactic Central Neck Dissection (pCND) groups.

\section{TT ALONE}

$n=25$

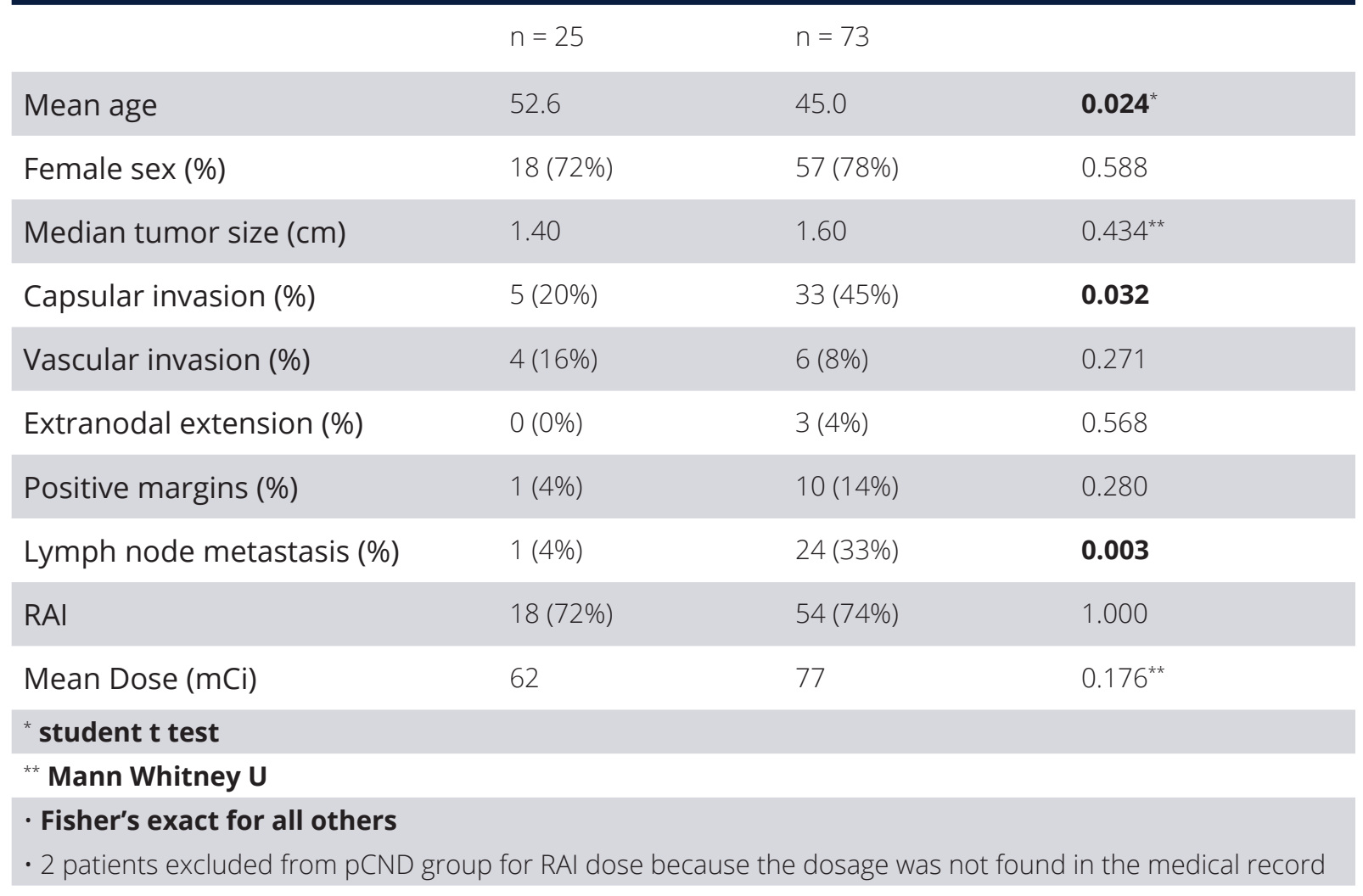

\section{TT WITH pCND p VALUE}

$n=73$

A subgroup analysis of TT with pCND patients was then performed comparing NO $(n=49)$ and N1a $(n=24)$ patients. Tumor characteristics and demographic data are presented in Table 2. The N1a cohort had 3 (12.5\%) patients with extranodal extension. Patients staged as N1a had a younger mean age (38.5 years vs 48.2 years, $p=0.006)$ and more vascular invasion (6 [25\%] vs $0[0 \%], p=0.001)$ than the N0 cohort. There were no statistically significant differences in sex $(p=0.765)$, tumor size $(p=0.255)$, capsular invasion ( $p=0.622$ ), or positive margins ( $p=0.720$ ) between the NO and N1a cohorts. The NO group received RAI therapy significantly less frequently than the N1a group (30 [61\%] vs 24 [100\%], $p<0.001)$. When comparing only patients receiving RAI therapy, the N1 a group did receive higher 131-I doses than the NO cohort (90 millicuries vs. 66 millicuries, $p=0.085$ ), but this did not meet statistical significance. 
TABLE 2. Clinicopathological variables and Radioactive lodine (RAI) between N0 and N1a subgroups within the Total Thyroidectomy (TT) with Prophylactic Central Neck Dissection (pCND) cohort.

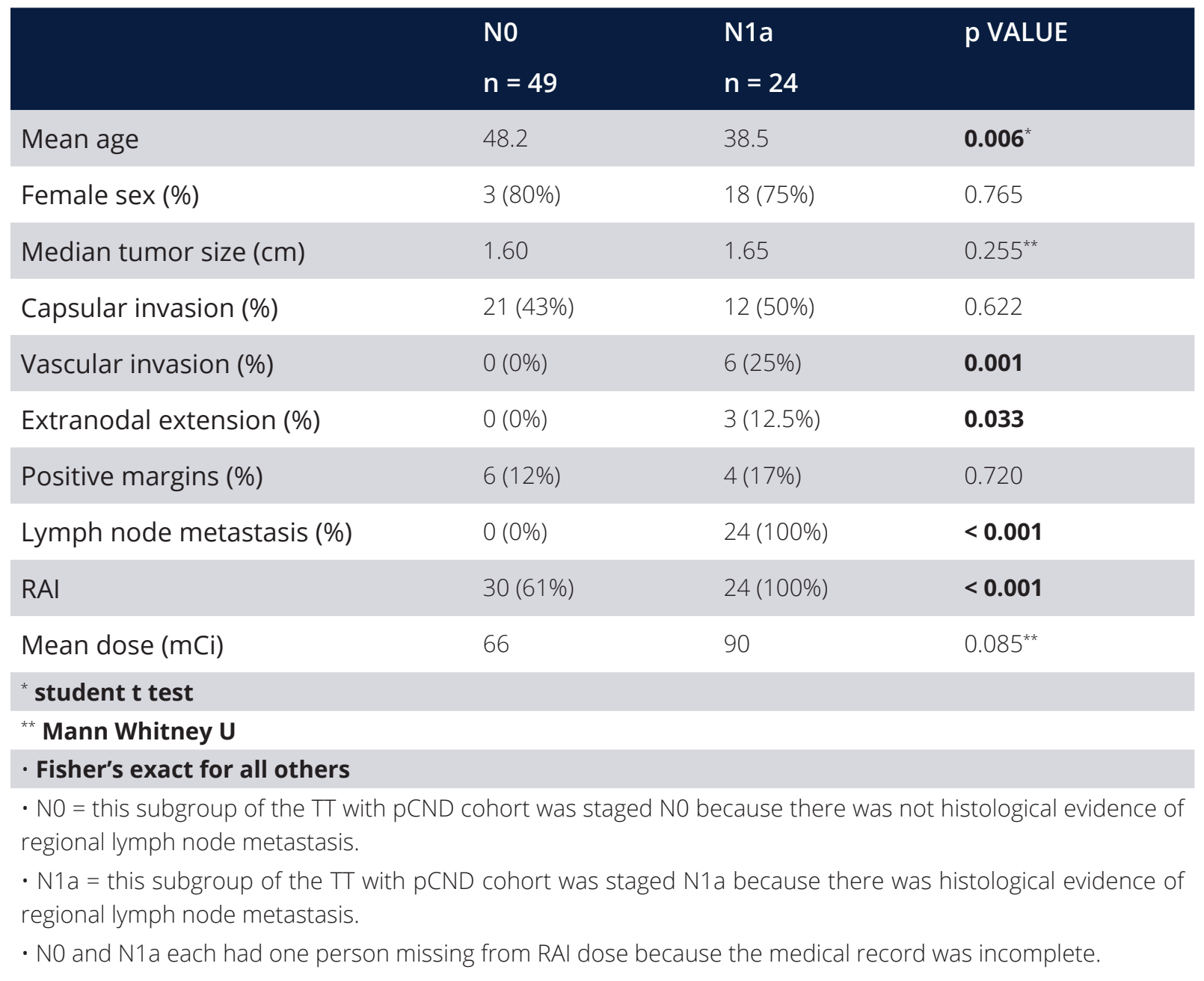

In regard to post-operative complications, no patients experienced permanent hypoparathyroidism or permanent vocal cord paralysis in our study in either the TT or TT with pCND groups.

\section{Discussion}

This study shows that TT with PCND does not increase RAI therapy when compared to the TT alone group. In fact, TT with pCND may ensure that a higher-staged group, N1a, receives RAl therapy while a lower staged group, NO, does not receive RAl therapy. All patients with N1a staged disease received RAI therapy while only $61 \%$ of NO patients received RAl therapy based upon the same ATA guidelines. TT with pCND stratified the NO patients as being a low-risk nodal stage, which likely contributed to them receiving RAI therapy less frequently. We do not believe that the statistically significant age difference between the TT and TT with pCND groups has clinical significance that will impact the outcome of this study. These results support our hypothesis that TT with pCND does not result in an overall increase in RAl therapy use but may allow for the use of RAl therapy in node positive (N1a) patients and avoidance in node negative (NO) patients.

Hughes et al did not address differences in the number of times RAl therapy was administered between the NO and N1a groups. Rather, they addressed dose differences between the groups 
in cases when RAl therapy was administered. ${ }^{16}$ Our dataset included a wider range of tumor size, particularly large tumors, than did Bonnet et al. ${ }^{15}$

As expected, lymph node metastasis was detected more often in patients undergoing TT with pCND. Only one incidental peri-isthmus lymph node metastasis was detected in the TT group. Notably, the upstaging of pCND patients to N1a did not lead to increased use of RAl therapy in the $\Pi T$ with pCND cohort. Previous studies have asserted that TT with PCND upstages PTC patients. ${ }^{10}$ While $T T$ with pCND does upstage some patients to $\mathrm{N} 1 \mathrm{a}$, this does not lead to a clinically meaningful increase in RAl therapy when looking at all patients who receive $\Pi T$ with pCND. This may be due, in part, to the fact that $T \pi$ with $P C N D$ also stages some patients as NO who are then less likely to receive RAI therapy. Previous studies have come to a similar conclusion: TT with pCND does not increase the risk of RAI therapy compared to TT alone. ${ }^{11,12}$

This study has several limitations. It is a retrospective review for a single tertiary care center with a highly selected group of patients, and it may not be truly representative of the surgical and RAI therapy algorithms at other institutions. Furthermore, an inherent selection bias exists as a surgeon chooses whether or not to perform a TT with pCND.

While future efforts should be made to address these limitations, this study is novel in that it adds to a body of work demonstrating that $T T$ with pCND does not result in an overall increase in RAl therapy use despite upstaging some patients to N1a and that $\Pi$ with pCND can be performed safely without an increased incidence of complications. We have shown that TT with pCND helps some NO patients avoid the risks associated with RAI therapy (sialadenitis, secondary malignancies) without increasing operative complications (hypocalcemia, laryngeal nerve injury). This conclusion is consistent with Viola et al's prospective randomized controlled study, which concluded that $T T$ with $\mathrm{pCND}$ reduced the use of RAI therapy. ${ }^{20}$
While the evidence for the safety of $T \mathrm{~T}$ with $\mathrm{pCND}$ varies, several other studies have shown that $\Pi T$ with $p C N D$ is a safe procedure with rates of permanent hypoparathyroidism and vocal cord paralysis due to recurrent laryngeal nerve injury similar to those experienced for TT alone. 3,16,21 In our own study, there were no instances of permanent hypoparathyroidism, symptomatic hypocalcemia, or hoarseness.

\section{Conclusion}

In summary, this study does not support the notion that TT with pCND upstages patients, leading to more RAl therapy. Rather, TT with pCND may more accurately direct the use of RAl therapy toward the higher-stage N1a group, while the lower-stage NO group does not receive RAl therapy as frequently. Future studies should include data from more institutions to understand this study's generalizability and use a large dataset to perform a multivariate analysis to further clarify the impact of TT with pCND on the use of RAI therapy.

\section{References}

1. Haugen BR, Alexander EK, Bible KC, et al. 2015 American Thyroid Association management guidelines for adult patients with thyroid nodules and differentiated thyroid cancer: the American Thyroid Association guidelines task force on thyroid nodules and differentiated thyroid cancer. Thyroid. 2016;26(1):1-133. doi:10.1089/ thy.2015.0020

2. Lang BH-H, Lo C-Y, Chan W-F, Lam K-Y, Wan K-Y. Prognostic factors in papillary and follicular thyroid carcinoma: their implications for cancer staging. Ann Surg Oncol. 2007;14(2):730-738. doi:10.1245/ s10434-006-9207-5

3. Wong K-P, Lang BH-H. The role of prophylactic central neck dissection in differentiated thyroid carcinoma: issues and controversies. J Oncol. 2011;2011:127929. doi:10.1155/2011/127929

4. Kaczka K, Fendler W, Borowiec M, Młynarski W, Celnik A, Pomorski L. Lymph node metastases in papillary thyroid cancer detected by quantitative real-time polymerase chain reaction for thyroglobulin and cytokeratine-19. Polish J Pathol. 2013;2:90-95. doi:10.5114/pjp.2013.36007 
5. Maxon HR, Smith HS. Radioiodine-131 in the diagnosis and treatment of metastatic well differentiated thyroid cancer. Endocrinol Metab Clin North Am. 1990;19(3):685-718.

6. Lee SL. Complications of radioactive iodine treatment of thyroid carcinoma. J Natl Compr Canc Netw. 2010;8(11):1277-86.

7. Ron E, Curtis R, Hoffman DA, Flannery JT. Multiple primary breast and thyroid cancer. $\mathrm{Br} J$ Cancer. 1984;49(1):87-92.

8. Carty SE, Cooper DS, Doherty GM, et al. Consensus statement on the terminology and classification of central neck dissection for thyroid cancer. Thyroid. 2009;19(11):1153-1158. doi:10.1089/thy.2009.0159

9. Ross DS, Litofsky D, Ain KB, et al. Recurrence after treatment of micropapillary thyroid cancer. Thyroid. 2009;19(10):1043-1048. doi:10.1089/thy.2008.0407

10. Randolph GW. Papillary cancer nodal surgery and the advisability of prophylactic central neck dissection: primum, non nocere. Surgery. 2010;148(6):1108-1112. doi:10.1016/j. surg.2010.09.035

11. Wang TS, Cheung K, Farrokhyar F, Roman SA, Sosa JA. A Meta-analysis of the effect of prophylactic central compartment neck dissection on locoregional recurrence rates in patients with papillary thyroid cancer. Ann Surg Oncol. 2013;20(11):3477-3483. doi:10.1245/s10434-013-3125-0

12. Sosa JA. Is routine prophylactic central neck dissection indicated for low-risk papillary thyroid cancer: can we determine cost-effectiveness if we are unsure about its effectiveness and safety. Surgery. 2013;154(6):1146-1147. doi:10.1016/j. surg.2013.06.026

13. Cisco RM, Shen WT, Gosnell JE. Extent of surgery for papillary thyroid cancer: preoperative imaging and role of prophylactic and therapeutic neck dissection. Curr Treat Options Oncol. 2012;13(1):1-10. doi:10.1007/s11864-011-0175-z

14. Barczyński M, Konturek A, Stopa M, Nowak W. Prophylactic central neck dissection for papillary thyroid cancer. Br J Surg. 2013;100(3):410-418. doi:10.1002/bjs.8985

15. Bonnet S, Hartl D, Leboulleux S, et al. Prophylactic lymph node dissection for papillary thyroid cancer less than $2 \mathrm{~cm}$ : implications for radioiodine treatment. J Clin Endocrinol Metab. 2009;94(4):1162-1167. doi:10.1210/jc.2008-1931

16. Hughes DT, White ML, Miller BS, Gauger PG, Burney RE, Doherty GM. Influence of prophylactic central lymph node dissection on postoperative thyroglobulin levels and radioiodine treatment in papillary thyroid cancer. Surgery. 2010;148(6):1100-1107. doi:10.1016/j.surg.2010.09.019

17. Cooper DS, Doherty GM, Haugen BR, et al. Management guidelines for patients with thyroid nodules and differentiated thyroid cancer. Thyroid. 2006;16(2):109-142. doi:10.1089/ thy.2006.16.109

18. Cooper DS, Doherty GM, Haugen BR, et al. Revised American Thyroid Association management guidelines for patients with thyroid nodules and differentiated thyroid cancer. 2009;19(11):1167-214. doi: 10.1089/thy.2009.0110

19. Carty SE, Cooper DS, Doherty GM, et al. Consensus statement on the terminology and classification of central neck dissection for thyroid cancer. 2009;19(11):1153-8. doi: 10.1089/ thy.2009.0159

20. Viola D, Materazzi G, Valerio L, et al. Prophylactic central compartment lymph node dissection in papillary thyroid carcinoma: clinical implications derived from the first prospective randomized controlled single institution study. J Clin Endocrinol Metab. 2015;100(4):1316-1324. doi:10.1210/ jc.2014-3825

21. Palestini N, Borasi A, Cestino L, Freddi $M$, Odasso C, Robecchi A. Is central neck dissection a safe procedure in the treatment of papillary thyroid cancer: our experience. Langenbeck's Arch Surg. 2008;393(5):693-698. doi:10.1007/ s00423-008-0360-0 